\title{
Experimental Study of Isolated Response Curves in a Two-degree-of-freedom Nonlinear System
}

\author{
T. Detroux ${ }^{1}$, J.P. Noël ${ }^{1}$, G. Kerschen ${ }^{1}$, L.N. Virgin ${ }^{2}$ \\ ${ }^{1}$ Space Structures and Systems Laboratory \\ Aerospace and Mechanical Engineering Department, University of Liège, Liège, Belgium \\ ${ }^{2}$ Nonlinear Dynamics Group \\ School of Engineering, Duke University, Durham, NC, USA
}

\begin{abstract}
In the present paper, the observation and characterization of isolated response curves (IRCs) are experimentally reported in the case of a nonlinear system consisting of two masses sliding on an horizontal guide. Transverse springs are attached to one mass to provide the nonlinear restoring force, and a harmonic motion of the complete system is imposed by prescribing the displacement of their supports. The existence of an IRC is related to a 3:1 internal resonance between the two modes of the system. The observed IRC is studied in detached and merged conditions using swept-sine excitations and system perturbations.
\end{abstract}

Keywords: isolated response curve, internal resonance, experimental observation, two-degree-of-freedom system, sinesweep excitation, perturbations.

\section{INTRODUCTION}

Isolated response curves (IRCs) are an intriguing feature of nonlinear dynamics. They correspond to closed loops of solutions emerging in nonlinear frequency responses and which are, by definition, detached from the main response branch ${ }^{[1]}$. IRCs may thus go easily undetected in the analysis of the forced response of a nonlinear system, whether it be numerically employing classical continuation techniques, or experimentally applying sine-sweep excitations. However, an increase in forcing amplitude may cause the merging of the main branch and the IRC, resulting in dramatic frequency and amplitude shifts of the resonance location. This renders IRCs potentially dangerous in practice for engineers designing systems likely to operate in nonlinear regimes of motion ${ }^{[2,3]}$.

In Ref. ${ }^{[4]}$, the authors investigated numerically a series of intrinsic features of IRCs, in particular their creation mechanism, the evolution of their bifurcations according to parameter variations and their basins of attraction. In the present paper, the observation and characterization of IRCs are experimentally reported in the case of a two-degree-of-freedom, base-excited mechanical system with nonlinear hardening springs. As it is conjectured that interactions between nonlinear modes underlie the existence of IRCs ${ }^{[5,6]}$, potential 3:1 internal resonances between the in-phase and out-of-phase modes of the system are specifically studied. Section 2 details the experimental setup of interest. In Section 3, the forced response of the setup to swept-sine excitations of various amplitudes is analyzed. The existence of an IRC is revealed through the sudden shift undergone by the resonance frequency of the in-phase mode. Perturbations are also applied to the system to observe the IRC when detached. Conclusions of the paper are summarized in Section 4. 


\section{AN EXPERIMENTAL TWO-DEGREE-OF-FREEDOM SYSTEM WITH HARDENING SPRINGS}

The experimental setup consists of two masses sliding on an horizontal guide, as shown in Figs. 1 (a) and (b). The masses are connected together and to the ground through extension springs, whose lengths and stiffnesses determine the static equilibrium of the system. Motion of the masses is recorded by means of uniaxial accelerometers. Two transverse bungee chords are attached to mass 1 (see Fig. 1 (b)) to provide a nonlinear restoring force in the direction of motion. The displacements of the transverse spring supports are prescribed to impart motion to the two masses using a Scotch yoke flywheel ${ }^{[7]}$. The Scotch yoke converts rotational motion into an unidirectional harmonic displacement $d=D \sin (\omega t)$. The frequency $\omega / 2 \pi$ was limited to $2.7 \mathrm{~Hz}$ in the present experimental test rig, and the amplitude $D$ was set manually, but could be accurately estimated.

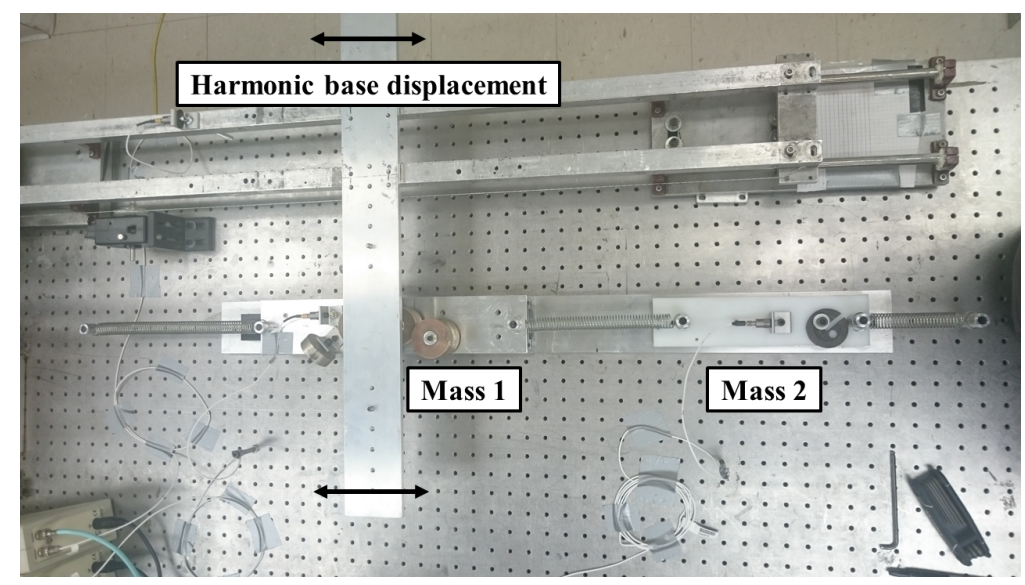

(a)

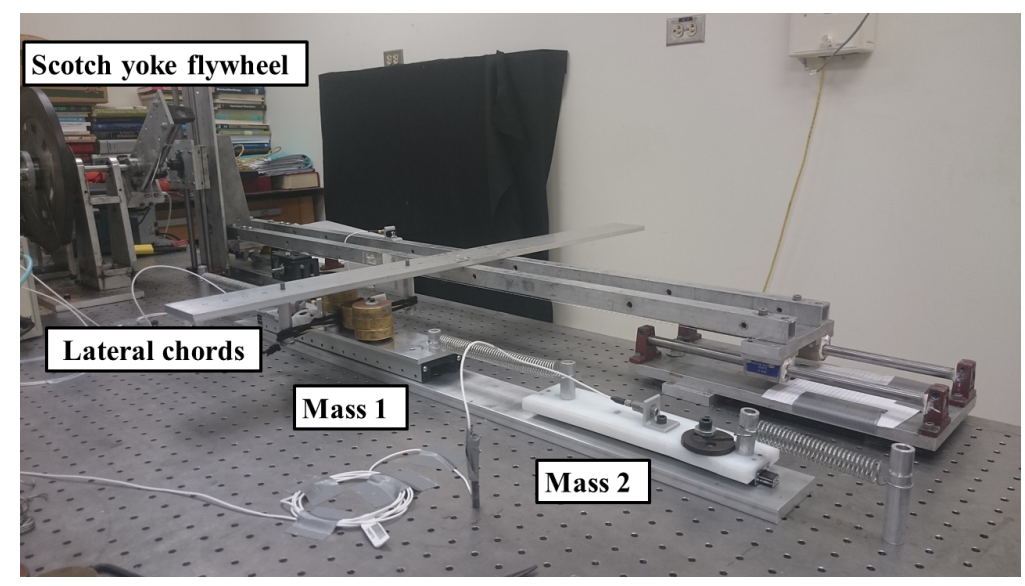

(b)

Figure 1: Experimental setup.

The restoring force in the system caused by the transverse bungee chords can be theoretically written as ${ }^{[4]}$

$$
F_{n l}(x)=2 k\left(1-\frac{\lambda}{\sqrt{1+(x / l)^{2}}}\right)
$$

where $x=x_{1}-d$ is the displacement of mass $1 x_{1}$ relative to the displacement of the base $d, k, l$ and $l_{0}$ denote the stiffness, 
length and natural length of the lateral springs, respectively, and $\lambda=l / l_{0}$ is the pre-stress parameter. The Taylor series expansion of Equation (1) around 0 reads

$$
F_{n l}(x)=(1-\lambda) x+\frac{\lambda}{2 l^{2}} x^{3}+R_{3}(x)
$$

Equation (2) indicates that the third-order term in the restoring force increases with the pre-stress parameter $\lambda$. In the present work, transverse bungee chords with no pre-stress, i.e. $\lambda=1$, were considered in order to facilitate the creation of IRCs, as suggested in Ref. ${ }^{[4]}$. Fig. 2 shows a qualitative estimate of the restoring force in the system calculated using the restoring force surface (RFS) method ${ }^{[8]}$, confirming the highly nonlinear behavior of the lateral spring connection.

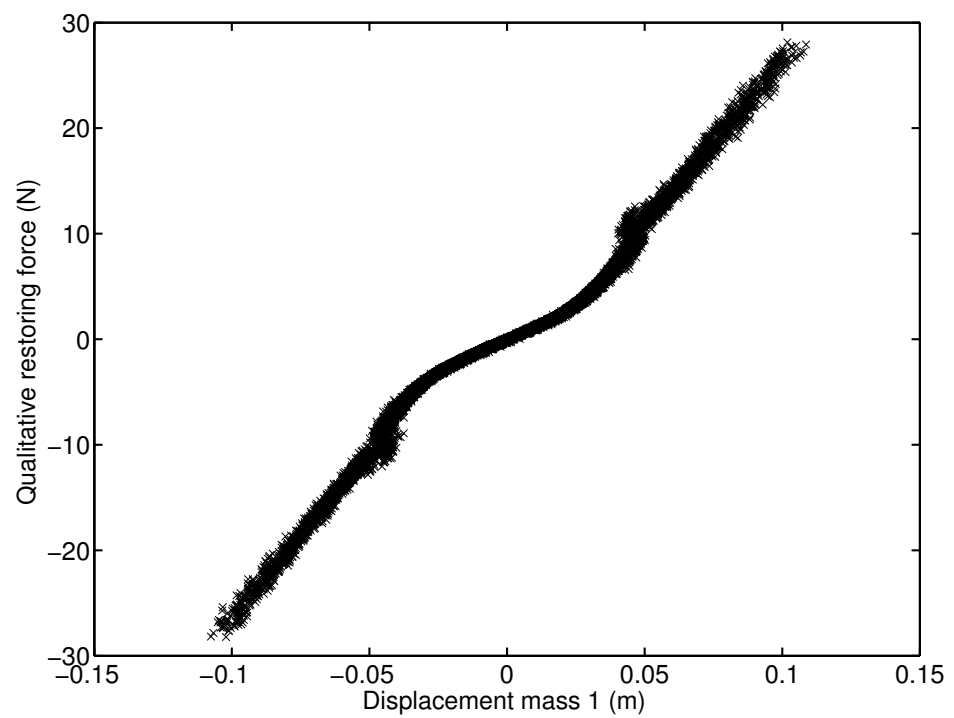

Figure 2: Qualitative restoring force of the lateral spring connection.

The estimation of the linear resonance frequencies of the setup is central in this study, as the potential existence of IRCs is conditional upon their ratio ${ }^{[4,5]}$. For that purpose, high-amplitude impacts were manually applied to the two masses, as illustrated in Fig. 3 (a). In this plot, three impacts on mass 2 are followed by three impacts on mass 1 . The resulting free decays can be analyzed to extract resonance frequencies. This is achieved in Fig. 3 (b) by plotting the wavelet transform of the acceleration measured on mass 1. The first three impacts mostly feature the second mode response around $6.45 \mathrm{~Hz}$. No amplitude-dependence is noticed for this mode proving its linearity. Conversely, the in-phase mode of the system, clearly observed during the three final impacts, exhibits a strong, hardening dependence upon the amplitude of motion. For sufficiently large response amplitudes, its third harmonic is seen to coincide with the second mode frequency, which is a necessary condition for the creation of an IRC via a 3:1 modal interaction ${ }^{[4]}$. Focusing on the tip of the free-decay behavior of mode 1 , its resonance frequency is estimated at $1.75 \mathrm{~Hz}$, yielding a ratio of 3.7 between the two mode linear frequencies. This favorable ratio was achieved by tuning the masses of the setup, which were eventually fixed to 3.67 (mass 1 ) and 0.45 (mass 2) $\mathrm{kg}$. 


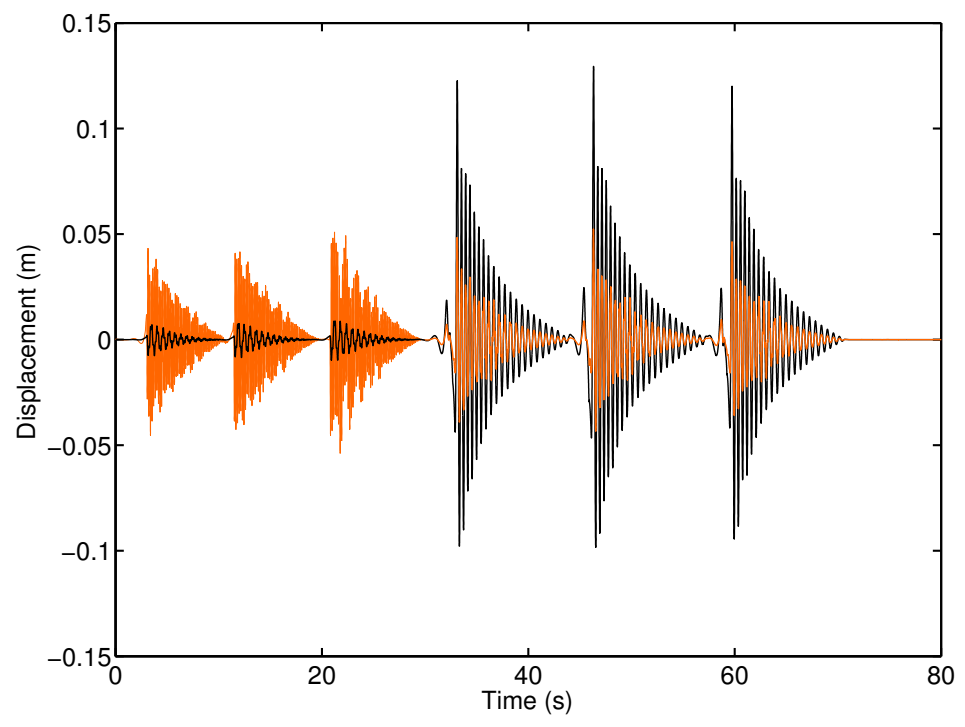

(a)

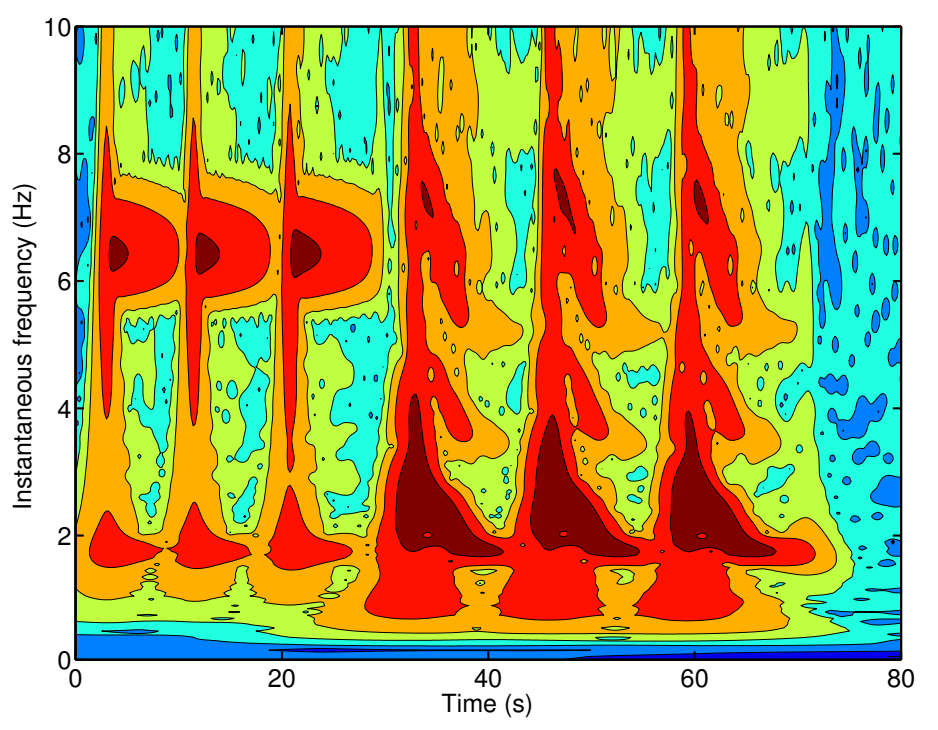

(b)

Figure 3: Free-decay analysis. (a) Displacements measured on mass 1 (in black) and mass 2 (in orange) in response to three impacts applied to mass 2 followed by three impacts applied to mass 1 . (b) Wavelet transform of mass 1 acceleration. 


\section{EXPERIMENTAL FORCED RESPONSES}

In this section, the forced response of the system to swept-sine base excitations of various amplitudes is studied. Throughout the section, a sweep rate of $0.13 \mathrm{~Hz} / \mathrm{min}$ is considered. Fig. 4 depicts the sweep-up (in black) and sweep-down (in blue) responses of mass 1 for a base amplitude of $5.9 \mathrm{~mm}$. Clear jumps down and up occur around $2.55 \mathrm{~Hz}$ and $1.8 \mathrm{~Hz}$, respectively. This large bi-stability region is a result of the strong nonlinearity in the system.

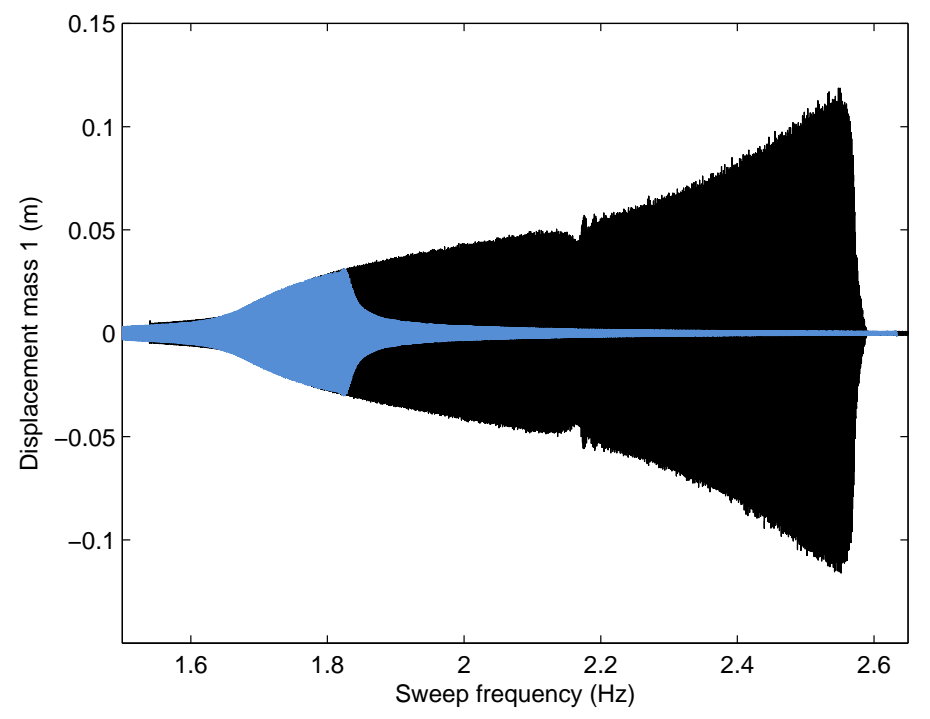

Figure 4: Response of mass 1 to a swept-sine excitation with an amplitude of $5.9 \mathrm{~mm}$. Sweep up (black); sweep down (blue).

In Fig. 5, the displacement of mass 1 in response to swept-sine forcing profiles of increasing amplitudes, namely $5,5.7,5.9$ and $6 \mathrm{~mm}$, is represented. A dramatic modification of the first mode resonance frequency from 2.15 to $2.55 \mathrm{~Hz}$, that is a nearly $20 \%$ rise, is observed when increasing the base amplitude from 5.7 to $5.9 \mathrm{~mm}$. This phenomenon, which also implies an increase of the response amplitude from 0.05 to $0.11 \mathrm{~m}$, is to be attributed to the merging of an IRC with the main response branch. The merging mechanism of an IRC is discussed in detail in Ref. ${ }^{[4]}$ through analysis and tracking of limit-point bifurcations.

It should be emphasized that the IRC merging in Fig. 5 occurs near $2.15 \mathrm{~Hz}$, that is, in the neighborhood of the $3: 1$ interaction between the in-phase and out-of-phase modes of the system. This merging frequency region is closely studied in Figs. 6 (a $-\mathrm{f}$ ). Figs. 6 (a) and (b) display the acceleration of mass 1 recorded for base displacements of 5.7 and 5.9 mm, respectively. In the former case, the IRC is detached, and the system jumps down to a low-amplitude solution around $2.15 \mathrm{~Hz}$. In the latter case, the IRC is merged, and a high-amplitude solution is continuously attainable when sweeping up the excitation frequency. The corresponding accelerations of mass 2 plotted in Figs. $6(c-d)$ show that it undergoes greater acceleration levels than mass 1 . Both mass accelerations also exhibit a strong beating behavior in the merging region in Figs. 6 (b) and (d). Finally, the existence of a 3:1 internal resonance in the system is highlighted in Figs. 6 (e) and (f), where the wavelet transform of Figs. 6 (c) and (d) is plotted, respectively. A strong third harmonic component is clearly seen to emerge in the vicinity of the out-of-phase mode frequency.

The possibility the realize a periodic solution on the detached IRC by perturbing the system is eventually investigated. For that purpose, a base excitation of $5 \mathrm{~mm}$ and $2.38 \mathrm{~Hz}$ is considered in Fig. 7. Initially, the system vibrates at low amplitude. A series of perturbations is applied until the IRC is reached and the motion stabilizes at high amplitude. A subsequent sweep up on the IRC permits to determine its domain of existence. A jump down around $2.47 \mathrm{~Hz}$ locates the right tip of its stable part. The excitation 


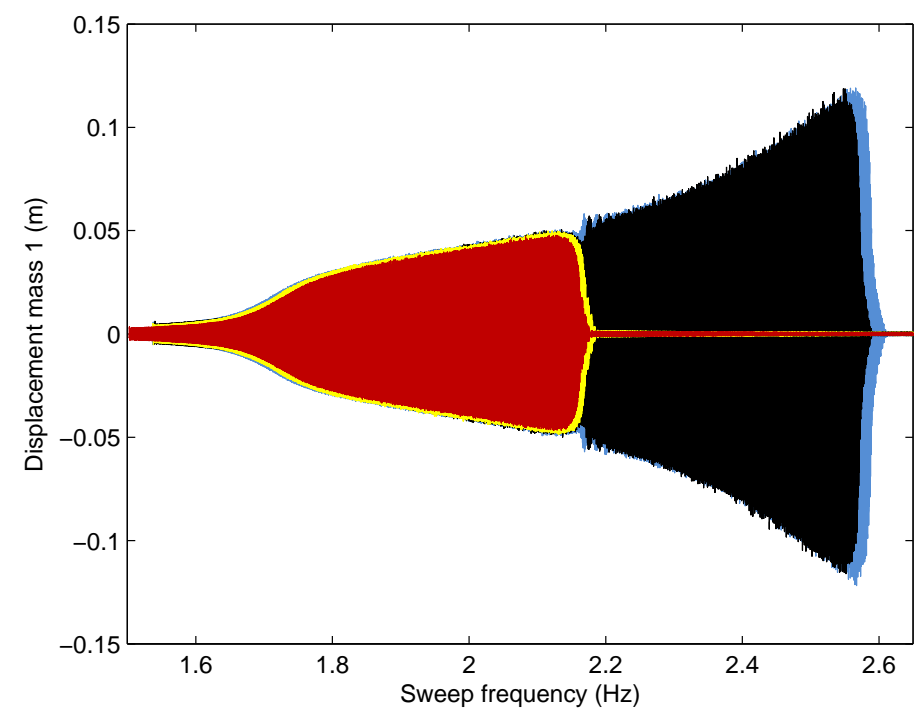

Figure 5: Response of mass 1 to swept-sine excitations of increasing amplitude: $5 \mathrm{~mm}$ (red); $5.7 \mathrm{~mm}$ (yellow); $5.9 \mathrm{~mm}$ (black); $6 \mathrm{~mm}$ (blue).

is then swept back to $2.38 \mathrm{~Hz}$, where new perturbations are applied. A sweep down along the stabilized IRC finally specifies the left tip of its stable part around $2.16 \mathrm{~Hz}$.

\section{CONCLUSIONS}

The objective of this paper was to investigate experimentally isolated response curves (IRCs) in a two-degree-of-freedom nonlinear system. More specifically, the paper intended to relate the existence of an IRC to a 3:1 internal resonance between the two modes of the system. To this end, swept-sine base excitations of various amplitudes were applied to the system. A dramatic modification of the first mode resonance frequency was noticed for small excitation amplitude increments. This frequency jump is the likely manifestation of the presence of an IRC, which expands and merges with the main frequency response as the excitation amplitude is increased. Nonetheless, the results reported in this paper deserve further investigation. In particular, a reliable numerical model of the setup should be developed in order to achieve a complete understanding of the observed phenomena. 


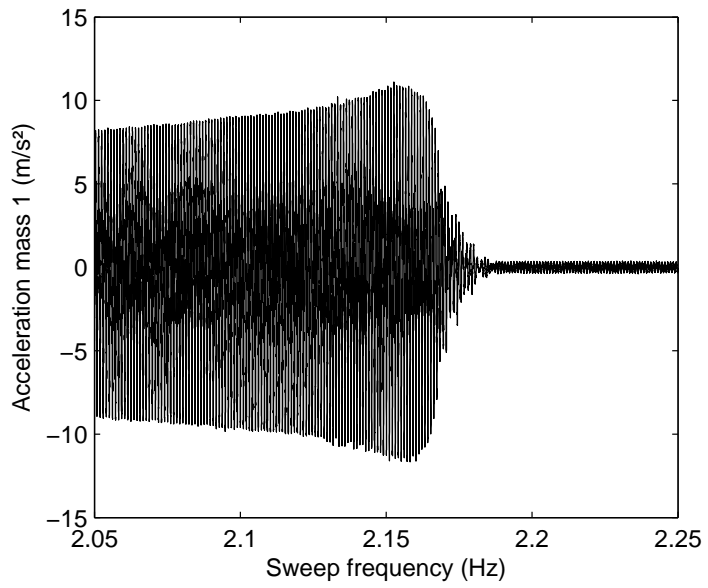

(a)

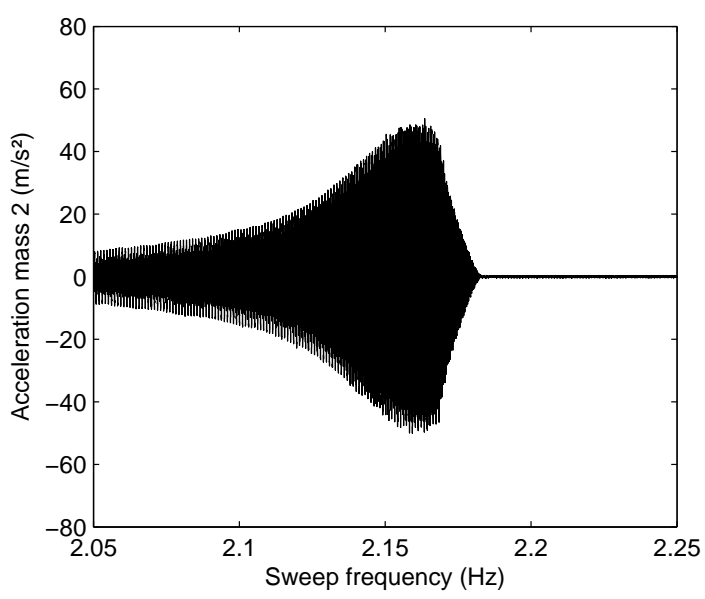

(c)

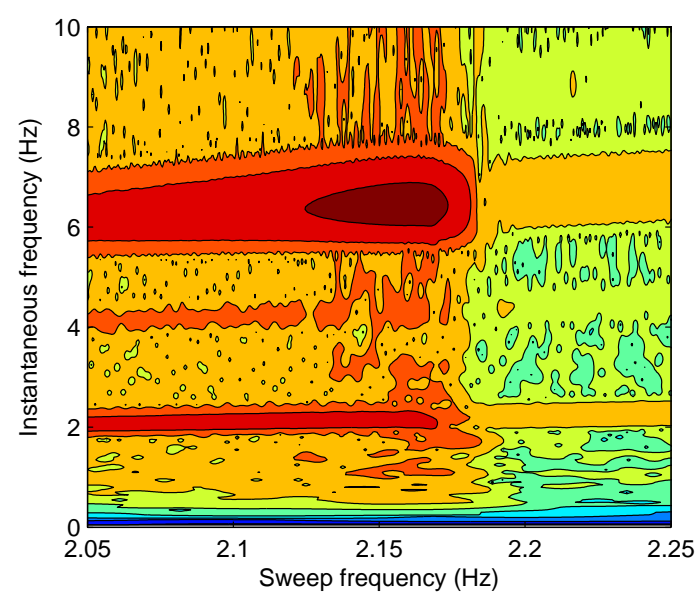

(e)

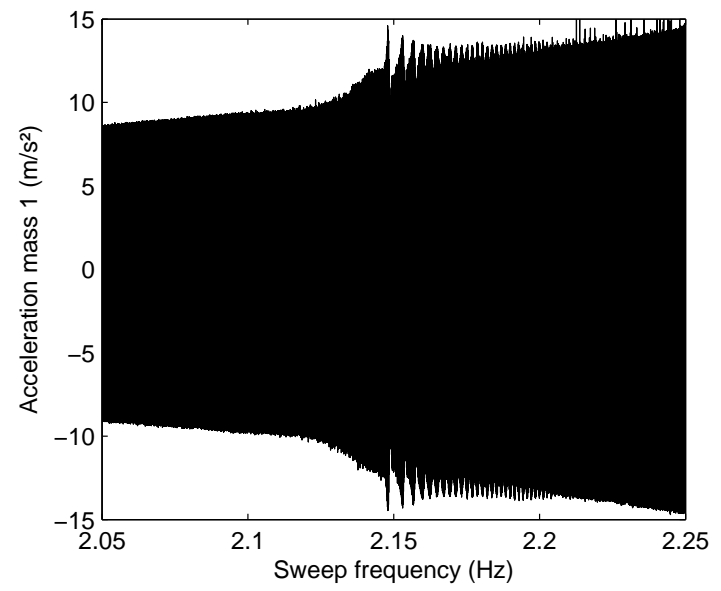

(b)

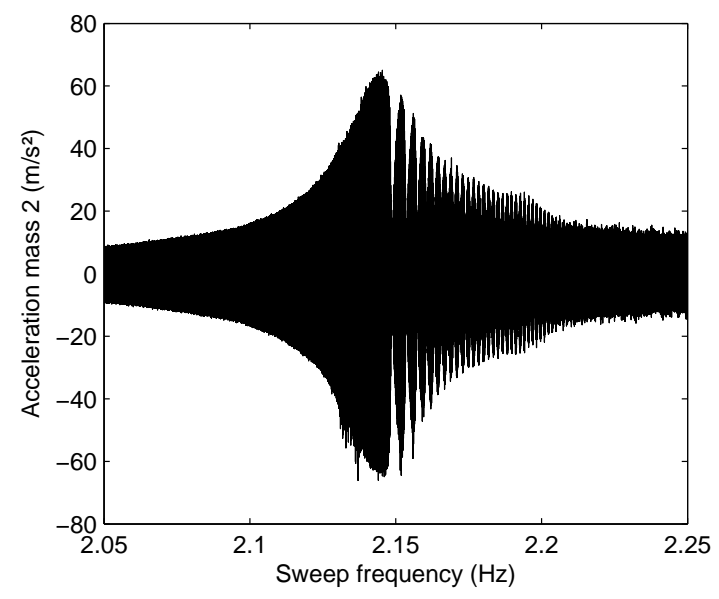

(d)

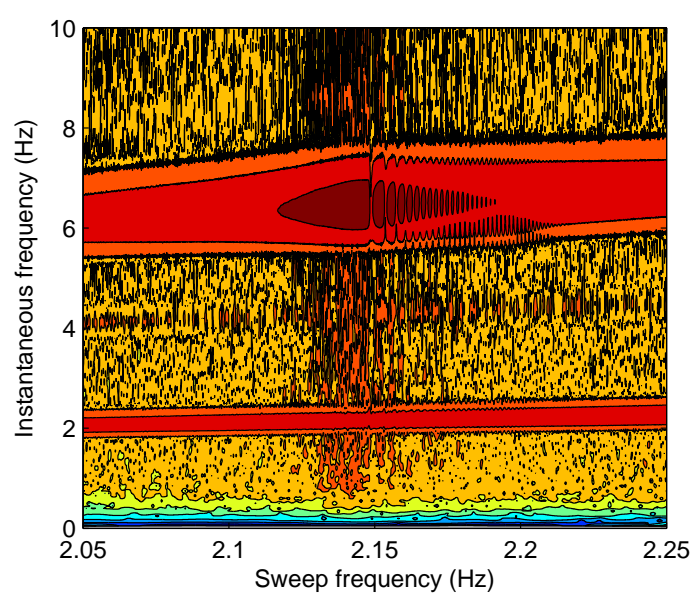

(f)

Figure 6: Merging of the IRC. Accelerations of mass 1 (a - b) and mass 2 (c - d) and wavelet transform of mass 2 acceleration $(e-f)$ in response to swept-sine excitations with amplitudes of $5.7 \mathbf{~ m m}$ (first column) and $5.9 \mathrm{~mm}$ (second column). 


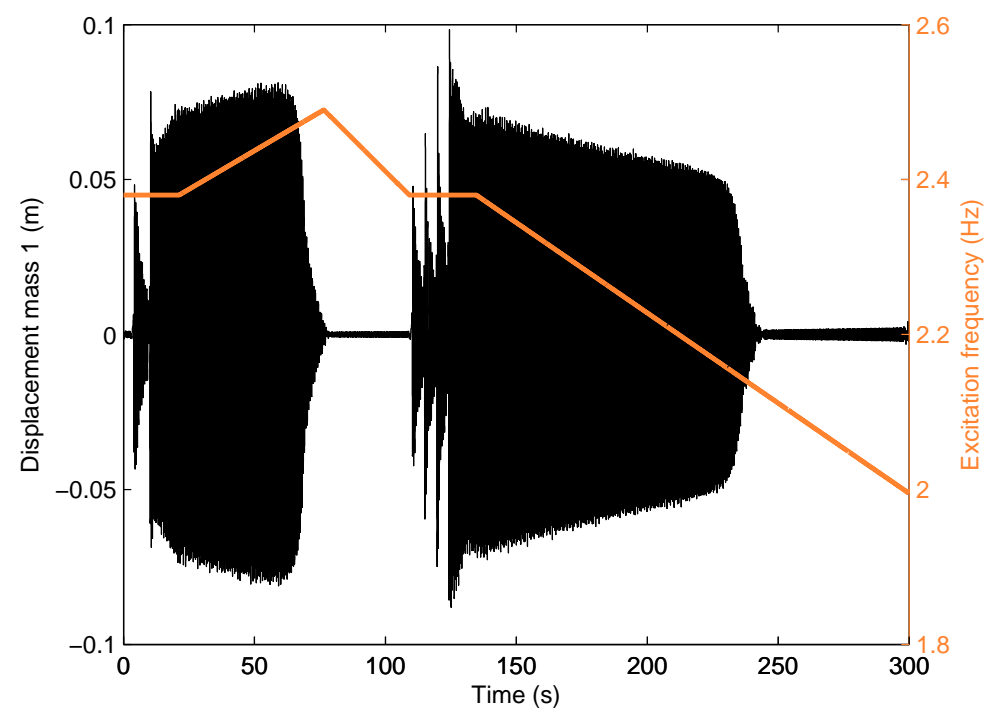

Figure 7: Realization of periodic solutions on the detached IRC by perturbations. The amplitude and initial frequency of the base excitation are $5 \mathbf{m m} 2.38 \mathrm{~Hz}$, respectively. The black and orange lines depict the displacement of mass $\mathbf{1}$ and the excitation frequency, respectively. 


\section{ACKNOWLEDGMENTS}

The authors T. Detroux and G. Kerschen would like to acknowledge the financial support of the European Union (ERC Starting Grant NoVib 307265). The author J.P. Noël is a Postdoctoral Researcher of the Fonds de la Recherche Scientifique - FNRS which is also gratefully acknowledged.

\section{References}

[1] Gatti, G., Brennan, M. and Kovacic, I., On the interactions of the responses at the resonance frequencies of a nonlinear two degrees-of-freedom system, Physica D, Vol. 239, pp. 591-599, 2010.

[2] Duan, C. and Singh, R., Isolated sub-harmonic resonance branch in the frequency response of an oscillator with slight asymmetry in the clearance, Journal of Sound and Vibration, Vol. 314, pp. 12-18, 2008.

[3] Alexander, N. and Schilder, F., Exploring the performance of a nonlinear tuned mass damper, Journal of Sound and Vibration, Vol. 319, pp. 445-462, 2009.

[4] Noël, J., Detroux, T., Kerschen, G. and Virgin, L., Isolated response curves in a base-excited, two-degree-of-freedom, nonlinear system, Proceedings of the ASME 2015 International Design Engineering Technical Conferences \& Computers and Information in Engineering Conference (IDETC/CIE), Boston, MA, 2015.

[5] Kuether, R., Renson, L., Detroux, T., Grappasonni, C., Kerschen, G. and Allen, M., Nonlinear normal modes, modal interactions and isolated resonance curves, Journal of Sound and Vibration, Vol. 351, pp. 299-310, 2015.

[6] Hill, T., Green, P., Cammarano, A. and Neild, S., Fast Bayesian identification of multi-mode systems using backbone curves, Journal of Sound and Vibration, Vol. In press, 2015.

[7] Virgin, L., Introduction to Experimental Nonlinear Dynamics: A Case Study in Mechanical Vibration, Cambridge University Press, Cambridge, United Kingdom, 2000.

[8] Noël, J., Renson, L. and Kerschen, G., Complex dynamics of a nonlinear aerospace structure: Experimental identification and modal interactions, Journal of Sound and Vibration, Vol. 333, pp. 2588-2607, 2014. 